\title{
The value of allogeneic and autologous hematopoietic stem cell transplantation in prognostically favorable acute myeloid leukemia with double mutant CEBPA
}

Schlenk, R F ; Taskesen, E ; van Norden, Y ; Krauter, J ; Ganser, A ; Bullinger, L ; Gaidzik, V I ; Paschka, P ; Corbacioglu, A ; Göhring, G ; Kündgen, A ; Held, G ; Götze, K ; Vellenga, E ; Kuball, J ; Schanz, U ; Passweg, J ; Pabst, Thomas ; Maertens, J ; Ossenkoppele, G J ; Delwel, R ; Döhner, H ; Cornelissen, J J ; Döhner, K ; Löwenberg, B

\begin{abstract}
The clinical value of allogeneic hematopoietic stem cell transplantation (alloHSCT) and autologous hematopoietic stem cell transplantation (autoHSCT) in the subtype of acute myeloid leukemia (AML) with double mutant CEBPA (CEBPAdm) has remained unsettled. Among 2983 patients analyzed for CEBPA mutational status (age 18-60 years) treated on 4 published Dutch-Belgian-Swiss HematoOncology Cooperative Group (HOVON/SAKK) and 3 German-Austrian AML Study Group (AMLSG) protocols (2 published, 1 registered, clinicaltrials.gov NCT00151255), 124 had AML with CEBPAdm and achieved first complete remission (CR1). Evaluation of the clinical impact of alloHSCT and autoHSCT vs chemotherapy was performed by addressing time dependency in the statistical analyses. Thirty-two patients proceeded to alloHSCT from a matched related $(\mathrm{MRD}, \mathrm{n}=29)$ or a matched unrelated donor (MUD, $\mathrm{n}=3$ ), 20 to autoHSCT in CR1 and 72 received chemotherapy. Relapse-free survival was significantly superior in patients receiving an alloHSCT or autoHSCT in CR1 as compared with chemotherapy $(\mathrm{P}<.001)$, whereas overall survival was not different $(\mathrm{P}<.12)$. Forty-five patients relapsed. Of 42 patients treated with reinduction therapy, 35 achieved a second CR (83\%) and most patients $(\mathrm{n}=33)$ received an alloHSCT MRD, $\mathrm{n}=11 ; \mathrm{MUD}, \mathrm{n}=19$; haplo-identical donor, $\mathrm{n}=3$ ). Survival of relapsed patients measured from date of relapse was $46 \%$ after 3 years. Adult AML patients with CEBPAdm benefit from alloHSCT and autoHSCT; relapsed patients still have a favorable outcome after reinduction followed by alloHSCT
\end{abstract}

DOI: https://doi.org/10.1182/blood-2013-05-503847

Posted at the Zurich Open Repository and Archive, University of Zurich

ZORA URL: https://doi.org/10.5167/uzh-85019

Journal Article

Published Version

Originally published at:

Schlenk, R F; Taskesen, E; van Norden, Y; Krauter, J; Ganser, A; Bullinger, L; Gaidzik, V I; Paschka, P; Corbacioglu, A; Göhring, G; Kündgen, A; Held, G; Götze, K; Vellenga, E; Kuball, J; Schanz, U; Passweg, J; Pabst, Thomas; Maertens, J; Ossenkoppele, G J; Delwel, R; Döhner, H; Cornelissen, J J; Döhner, K; Löwenberg, B (2013). The value of allogeneic and autologous hematopoietic stem cell transplantation in prognostically favorable acute myeloid leukemia with double mutant CEBPA. Blood, 9(122):1576-1582. DOI: https://doi.org/10.1182/blood-2013-05-503847 


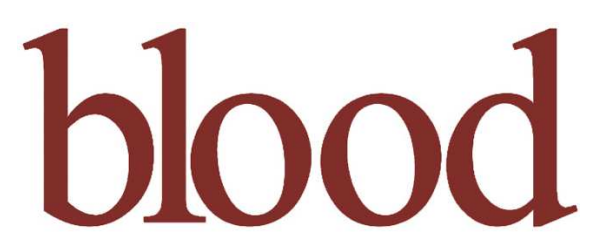

2013 122: 1576-1582

Prepublished online July 17, 2013;

doi:10.1182/blood-2013-05-503847

\section{The value of allogeneic and autologous hematopoietic stem cell transplantation in prognostically favorable acute myeloid leukemia with double mutant CEBPA}

Richard F. Schlenk, Erdogan Taskesen, Yvette van Norden, Jürgen Krauter, Arnold Ganser, Lars Bullinger, Verena I. Gaidzik, Peter Paschka, Andrea Corbacioglu, Gudrun Göhring, Andrea Kündgen, Gerhard Held, Katharina Götze, Edo Vellenga, Juergen Kuball, Urs Schanz, Jakob Passweg, Thomas Pabst, Johan Maertens, Gert J. Ossenkoppele, Ruud Delwel, Hartmut Döhner, Jan J. Cornelissen, Konstanze Döhner and Bob Löwenberg

Updated information and services can be found at: http://bloodjournal.hematologylibrary.org/content/122/9/1576.full.html

Articles on similar topics can be found in the following Blood collections

Clinical Trials and Observations (3781 articles)

Free Research Articles (2028 articles)

Myeloid Neoplasia (1066 articles)

Transplantation (1927 articles)

Information about reproducing this article in parts or in its entirety may be found online at: http://bloodjournal.hematologylibrary.org/site/misc/rights.xhtml\#repub_requests

Information about ordering reprints may be found online at:

http://bloodjournal.hematologylibrary.org/site/misc/rights.xhtml\#reprints

Information about subscriptions and ASH membership may be found online at:

http://bloodjournal.hematologylibrary.org/site/subscriptions/index.xhtml

Blood (print ISSN 0006-4971, online ISSN 1528-0020), is published weekly by the American Society of Hematology, 2021 L St, NW, Suite 900,

Washington DC 20036.

Copyright 2011 by The American Society of Hematology; all rights reserved.

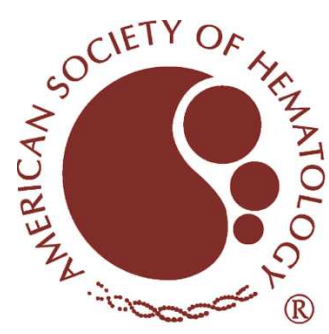




\title{
CLINICAL TRIALS AND OBSERVATIONS
}

\section{The value of allogeneic and autologous hematopoietic stem cell transplantation in prognostically favorable acute myeloid leukemia with double mutant $C E B P A$}

\author{
Richard F. Schlenk, ${ }^{1}$ Erdogan Taskesen, ${ }^{2}$ Yvette van Norden, ${ }^{3}$ Jürgen Krauter, ${ }^{4}$ Arnold Ganser, ${ }^{4}$ Lars Bullinger, ${ }^{1}$ \\ Verena I. Gaidzik, ${ }^{1}$ Peter Paschka, ${ }^{1}$ Andrea Corbacioglu, ${ }^{1}$ Gudrun Göhring, ${ }^{5}$ Andrea Kündgen, ${ }^{6}$ Gerhard Held, ${ }^{7}$ \\ Katharina Götze, ${ }^{8}$ Edo Vellenga, ${ }^{9}$ Juergen Kuball, ${ }^{10}$ Urs Schanz, ${ }^{11}$ Jakob Passweg, ${ }^{12}$ Thomas Pabst, ${ }^{13}$ Johan Maertens, ${ }^{14}$ \\ Gert J. Ossenkoppele, ${ }^{15}$ Ruud Delwel, ${ }^{2}$ Hartmut Döhner, ${ }^{1}$ Jan J. Cornelissen, ${ }^{2}$ Konstanze Döhner, ${ }^{1}$ and Bob Löwenberg ${ }^{2}$ \\ ${ }^{1}$ Department of Internal Medicine III, University of Ulm, Ulm, Germany; ${ }^{2}$ Department of Hematology and ${ }^{3}$ Department of Trials and Statistics, Erasmus \\ University Medical Center, Rotterdam, The Netherlands; ${ }^{4}$ Department of Hematology, Hemostasis, Oncology and Stem Cell Transplantation and ${ }^{5}$ Institute \\ of Cell and Molecular Pathology, Hannover Medical School, Hannover, Germany; ${ }^{6}$ Department of Hematology, Oncology and Clinical Immunology, \\ Heinrich-Heine-University, Düsseldorf, Germany; ${ }^{7}$ Department of Internal Medicine, University of Homburg, Homburg, Germany; ${ }^{8}$ Department of Internal \\ Medicine III, University of Munich, Munich, Germany; ${ }^{9}$ Department of Experimental Hematology, University Medical Center Groningen, University of \\ Groningen, The Netherlands; ${ }^{10}$ Department of Hematology, University Medical Center Utrecht, Utrecht, The Netherlands; ${ }^{11}$ Division of Hematology, \\ University Hospital, Zürich, Switzerland; ${ }^{12}$ Department of Hematology, University Hospital, Basel, Switzerland; ${ }^{13}$ Department of Hematology, University \\ Hospital, Bern, Switzerland; ${ }^{14}$ Department of Hematology, University Hospital Gasthuisberg, Leuven, Belgium; and ${ }^{15}$ Division of Hematology, VU University \\ Medical Center, Amsterdam, The Netherlands
}

\section{Key Points}

- In AML with bialleleic CEBPAmut relapse-free survival was improved by allogeneic and autologous hematopoietic stem cell transplantation.

- In relapsed patients second complete remission rate was high and survival was transplantation. favorable after an allogeneic

The clinical value of allogeneic hematopoietic stem cell transplantation (alloHSCT) and autologous hematopoietic stem cell transplantation (autoHSCT) in the subtype of acute myeloid leukemia (AML) with double mutant CEBPA (CEBPAdm) has remained unsettled. Among 2983 patients analyzed for CEBPA mutational status (age 18-60 years) treated on 4 published Dutch-Belgian-Swiss Hemato-Oncology Cooperative Group (HOVON/SAKK) and 3 German-Austrian AML Study Group (AMLSG) protocols (2 published, 1 registered, clinicaltrials.gov NCT00151255), 124 had AML with CEBPAdm and achieved first complete remission (CR1). Evaluation of the clinical impact of alloHSCT and autoHSCT vs chemotherapy was performed by addressing time dependency in the statistical analyses. Thirty-two patients proceeded to alloHSCT from a matched related (MRD, $n=29$ ) or a matched unrelated donor (MUD, $n=3$ ), 20 to autoHSCT in CR1 and 72 received chemotherapy. Relapse-free survival was significantly superior in patients receiving an alloHSCT or autoHSCT in CR1 as compared with chemotherapy $(P<.001)$, whereas overall survival was not different $(P<.12)$. Forty-five patients relapsed. Of 42 patients treated with reinduction therapy, 35 achieved a second CR $(83 \%)$ and most patients $(n=33)$ received an alloHSCT MRD, $n=11$; MUD, $n=19$; haplo-identical donor, $n=3$ ). Survival of relapsed patients measured from date of relapse was $46 \%$ after 3 years. Adult AML patients with CEBPAdm benefit from alloHSCT and autoHSCT; relapsed patients still have a favorable outcome after reinduction followed by alloHSCT. (Blood. 2013;122(9):1576-1582)

\section{Introduction}

Acute myeloid leukemia (AML) with mutated CCAAT/enhancer binding protein $\alpha(C E B P A)$ gene represents a provisional disease entity in the current World Health Organization (WHO) classification in the category "AML with recurrent genetic abnormalities." However, multiple studies demonstrated that AML with double mutant CEBPA (CEBPAdm) could be clearly distinguished from AML with single mutant $C E B P A$ with respect to biological and prognostic features. ${ }^{3-8}$ In the majority of AML with $C E B P A \mathrm{dm}$, one allele is affected by an $N$-terminal mutation and the second allele carries the mutation in the C-terminus (bZIP), whereas in AML with a single mutant $C E B P A$, mutations occur either in the $\mathrm{N}$-terminus or in the C-terminus of the gene. ${ }^{9}$ The previously shown favorable impact of mutant $C E B P A$ in various independent comprehensive studies on prognosis ${ }^{10}$ has more recently been specifically related to the subtype of AML with CEBPAdm. ${ }^{3-8}$

The incidence of AML with mutated (single and double) $C E B P A$ ranges from $7.5 \%$ to $11 \%$ of all AML patients and from about $13 \%$ to $18 \%$ in AML exhibiting a normal karyotype..$^{3-8,10-12}$ Furthermore, the incidence of AML with mutated CEBPA in patients who are $>60$ years range from $8.5 \%{ }^{13}$ to $18 \% .^{14}$
Submitted May 19, 2013; accepted June 28, 2013. Prepublished online as Blood First Edition paper, July 17, 2013; DOI 10.1182/blood-2013-05-503847.

R.F.S. and E.T. contributed equally to this work.

The online version of this article contains a data supplement.
The publication costs of this article were defrayed in part by page charge payment. Therefore, and solely to indicate this fact, this article is hereby marked "advertisement" in accordance with 18 USC section 1734. 
Young and middle aged adults (age 18-60 years) with AML and mutated $C E B P A$ and especially those with $C E B P A \mathrm{dm}$ have a comparatively high probability of achieving a complete remission after standard " $7+3$ " induction therapy with remission rates exceeding 90\%. ${ }^{6,8}$ Treatment outcome data revealed a favorable prognosis with an overall survival (OS) after 5 years ranging between $50 \%$ and $70 \%,{ }^{3-8}$ including different types of consolidation therapy with intensive chemotherapy, autologous hematopoietic stem cell transplantation (autoHSCT), and allogeneic hematopoietic stem cell transplantation (alloHSCT). However, relapse still remains the major cause of treatment failure that mainly occurs within the first 2 years after achieving a complete remission (CR). This has for instance raised the question whether autoHSCT and alloHSCT in (CR1) should be recommended in patients with this genetic abnormality. So far, analyses according to the type of postremission treatment in CEBPAdm AML patients have not become available mainly due to limited patient numbers precluding informative statistical analyses. Thus, it still remains unclear whether the favorable prognosis of AML with CEBPAdm can be attributed to the mutation itself irrespective of the type of applied postremission therapy (ie, prognostic marker) or whether the favorable prognosis is the result of a high rate of cure after autoHSCT and alloHSCT in $\mathrm{CR} 1$ and after relapse (ie, predictive marker). Informative insight into these factors could be of direct clinical relevance, as it may guide treatment decisions on the application of autoHSCT and alloHSCT already in CR1 or alternatively to hold back on these approaches and reserve the option especially of an alloHSCT as salvage only in relapsed patients. To address this question, the Dutch-Belgian-Swiss Hemato-Oncology Cooperative Group (HOVON/SAKK) and the German-Austrian AML Study Group (AMLSG), as leukemia cooperative groups, performed a joint effort on an individual-patient based meta-analysis focusing on the AML CEBPAdm subtype in CR1. The aim was to evaluate different postremission strategies with a major focus on the comparison between alloHSCT, autoHSCT, and intensive chemotherapy in a large series of CEBPAdm AML patients in CR1. Furthermore, with an integrated approach, we also included treatment after relapse and its impact on outcome.

\section{Patients and methods}

\section{Patients and treatment}

All patients included in this study were recruited within 2 major leukemia cohorts. AML patients from cohort I $(\mathrm{n}=3450)$ were enrolled in the HOVON/SAKK trials HOVON04(A), HOVON29/SAKK30/95, ${ }^{15-17}$ HOVON42(A)/SAKK30/00, and HOVON92/SAKK30/08 $8^{16-18}$ (information on the trials is avaibale at www.hovon.nl). Patients received 2 successive cycles of anthracycline-cytarabine- and amsacrine-cytarabinebased remission induction chemotherapy and, subsequently, in CR1 consolidation chemotherapy, autoHSCT after myeloablative therapy according to a randomization against chemotherapy and depending on an adequate stem cell collection, ${ }^{18}$ or alloHSCT after mainly myeloablative conditioning depending on the availability of a matched related donor (MRD).

Cohort II $(n=2274)$ comprised patients who were enrolled in the AMLSG trials AML HD93, ${ }^{19}$ AML HD98A, ${ }^{20}$ and AMLSG 07-04 (ClinicalTrials.gov Identifier: NCT00151242). Consistently throughout all AMLSG trials, patients with AML exhibiting an intermediate-risk karyotype with mutant $C E B P A$ were intended to receive a double induction therapy with idarubicin, cytarabine, and etoposide, and repetitive cycles of high-dose cytarabine-based consolidation therapy, or, if an HLA-matched family donor was available, an allogeneic hematopoietic stem cell transplantation (HSCT) after a myeloablative conditioning regimen.

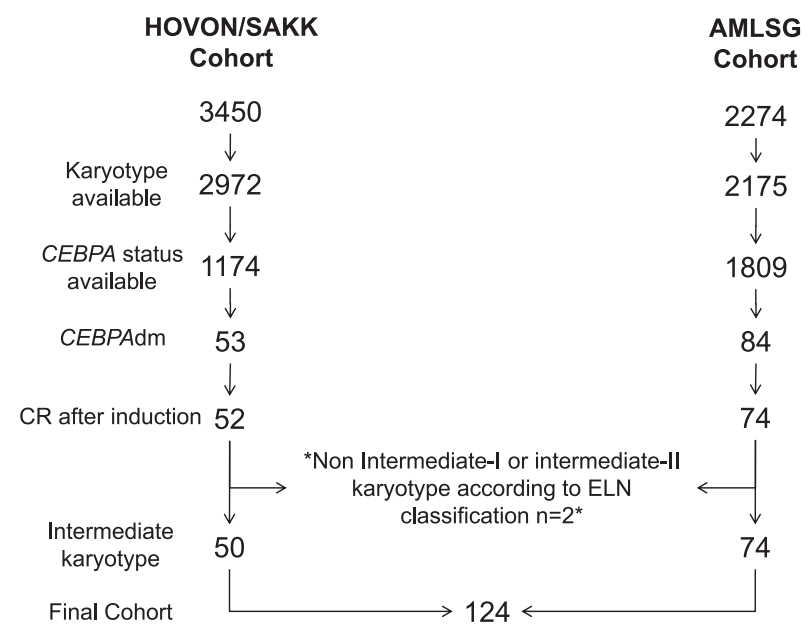

*i) complex karyotype including $-5 / 7(q)-$, ii) inv(16)(p13q22)

Figure 1. Flowchart on patient selection. Number of patients according to each selection step.

Patients were selected from the total cohort if they fulfilled all 3 of the following criteria: (1) normal karyotype or intermediate-risk karyotype according to EuropeanLeukemiaNet (ELN) $\mathrm{criteria}^{2}{ }^{2}$ (2) CEBPAdm; and (3) CR after induction therapy. The selection process is illustrated in Figure 1. In $90 \%$ (5147 of 5724) of the patients, information on cytogenetics was available. In these 5147 patients, the CEBPA mutation status was available in $2983(58 \%)$; of those, 137 exhibited a CEBPAdm in the context of a normal karyotype or intermediate-risk cytogenetics (4.6\%). There were 124 patients who achieved a CR1 after induction therapy within the different protocols $(90.5 \% \mathrm{CR}$ rate) who were included into this study.

All patients provided written informed consent in accordance with the Declaration of Helsinki. All trials were approved by the institutional review boards. CEBPA mutational status was identified by denaturing highperformance liquid chromatography, polymerase chain reaction amplification followed by direct sequencing or fragment-length analysis (GeneScan) and subsequent sequence analysis in any positive cases. ${ }^{4,5}$ Cytogenetics and molecular analyses were performed as described before. ${ }^{8,10,21-23}$

\section{Statistical analysis}

The definition of $\mathrm{CR}$ and survival end points such as OS, cumulative incidence of relapse (CIR), and death in CR (CID), as well as relapse-free survival (RFS) were based on the recommended consensus criteria. ${ }^{2}$ Actuarial estimates were used for the assessment of median follow-up for survival. Patient characteristics were compared by the Kruskal-Wallis test (continuous variables) and the Fisher's exact test (categorical variables). CIR and CID were analyzed according to the method of Gray. ${ }^{24}$ To address the time dependence of the variables alloHSCT and autoHSCT, the graphical representation, according to the method of Simon and Makuch ${ }^{25}$ was used, as well as the Mantel-Byar test, as appropriated statistical approach in univariable analyses. ${ }^{26}$ For multivariable analyses, an extended Cox regression model was used according to the method of Andersen and Gill. ${ }^{27}$ For all analyses, $P \leqslant .05$ was considered statistically significant. All statistical analyses were performed using the statistical software Stata Statistical Software (release 12).

\section{Results}

\section{Demographics and clinical baseline characteristics of the study population}

In this cooperative individual patient data meta-analysis, 124 $C E B P A \mathrm{dm}$ AML patients were included with normal karyotype or intermediate-risk cytogenetics (ages between 18 and 60 years and 
Table 1. Clinical and genetic characteristics of the total cohort and according to applied postremission therapy

\begin{tabular}{|c|c|c|c|c|c|}
\hline & $\begin{array}{l}\text { Total } \\
\text { cohort }\end{array}$ & AlloHSCT & AutoHSCT & no HSCT & $P$ value \\
\hline Characteristics & $n=124$ & $\mathrm{n}=32$ & $n=20$ & $\mathrm{n}=72$ & \\
\hline \multicolumn{6}{|l|}{ Age, y } \\
\hline Median (range) & $44(16-60)$ & 40 & 41 & 45 & 12 \\
\hline \multicolumn{6}{|l|}{ Sex, no. (\%) } \\
\hline Male & $66(53 \%)$ & $21(66 \%)$ & $12(60 \%)$ & $33(46 \%)$ & .15 \\
\hline Female & $58(47 \%)$ & $11(34 \%)$ & $8(40 \%)$ & 39 (54\%) & \\
\hline \multicolumn{6}{|l|}{ WBC, $\times 10^{9} / I$} \\
\hline Median (range) & $32(2-248)$ & $16(2-174)$ & $36(3-157)$ & $34(2-248)$ & .23 \\
\hline Missing & 1 & & & 1 & \\
\hline \multicolumn{6}{|l|}{ Platelets, $\times 10^{9} / I$} \\
\hline Median (range) & $41(4-319)$ & $39(11.282)$ & $47(10-115)$ & $40(4-319)$ & .42 \\
\hline Missing & 3 & & & 3 & \\
\hline \multicolumn{6}{|l|}{$\begin{array}{r}\text { Bone marrow } \\
\text { blasts, (\%) }\end{array}$} \\
\hline Median (range) & $75(7-100)$ & $71(7-99)$ & 77 (31-99) & $75(25-100)$ & .54 \\
\hline Missing & 5 & 1 & & 4 & \\
\hline \multicolumn{6}{|l|}{$\begin{array}{l}\text { Type of AML, } \\
\text { no. (\%) }\end{array}$} \\
\hline \multicolumn{6}{|l|}{ De novo AML } \\
\hline MO & $6(5 \%)$ & $1(3 \%)$ & & $5(7 \%)$ & 24 \\
\hline M1 & 45 (36\%) & $10(31 \%)$ & $9(45 \%)$ & $26(36 \%)$ & \\
\hline M2 & $54(44 \%)$ & $16(50 \%)$ & $6(30 \%)$ & 32 (44\%) & \\
\hline M4 & $6(5 \%)$ & $2(6 \%)$ & $2(10 \%)$ & $2(3 \%)$ & \\
\hline M5 & $2(2 \%)$ & $1(3 \%)$ & $1(5 \%)$ & & \\
\hline M6 & $1(1 \%)$ & & $1(5 \%)$ & & \\
\hline Unclassified & $1(1 \%)$ & & & $1(1 \%)$ & \\
\hline Missing & $9(7 \%)$ & $2(6 \%)$ & $1(5 \%)$ & $6(9 \%)$ & \\
\hline \multicolumn{6}{|l|}{$\begin{array}{c}\text { Cytogenetics, } \\
\text { no, (\%) }\end{array}$} \\
\hline $\begin{array}{l}\text { Normal } \\
\text { karyotype }\end{array}$ & $92(74 \%)$ & $21(66 \%)$ & $14(70 \%)$ & $57(79 \%)$ & .32 \\
\hline \multicolumn{6}{|l|}{$\begin{array}{l}\text { Molecular } \\
\text { characteristics } \\
\text { no., (\%) }\end{array}$} \\
\hline $\begin{array}{l}\text { FLT3-internal } \\
\text { tandem } \\
\text { duplications }\end{array}$ & $11(9 \%)$ & $5(16 \%)$ & $1(5 \%)$ & $5(7 \%)$ & .38 \\
\hline NPM1 & $2(2 \%)$ & $1(3 \%)$ & & $1(1 \%)$ & .67 \\
\hline Missing & 1 & & 1 & & \\
\hline
\end{tabular}

Subheadings under "de novo AML" refer to French American British classification subtypes.

WBC, white blood cell count; FLT3-ITD, FLT3 internal tandem duplication; neg, negative; NPM1, nucleophosmin 1; WBC, white blood cell count; wt, wild-type.

CR1 after induction therapy). The patients were selected from the total study population treated in the HOVON/SAKK and AMLSG prospective multicenter clinical trials recruited between 1987 and 2009 (Figure 1). Baseline characteristics and demographics for the total cohort are shown in Table 1.

No significant difference in OS was seen between the HOVON/ SAKK and the AMLSG CEBPAdm patient cohorts ( $\mathrm{n}=50 \mathrm{vs}$ $\mathrm{n}=74$, Cox test $P=.36$ ); molecular and clinical variables were comparable between the HOVON/SAKK and the AMLSG CEBPAdm patient cohorts with the exception of platelet counts $(P=.02$, lower in AMLSG) and bone marrow blasts $(P<.0001$, lower in HOVON/ SAKK).

\section{Postremission therapy, CIR, and CID in CEBPAdm patients}

Distribution of postremission treatment modalities in the 124 patients was as follows: alloHSCT, $\mathrm{n}=32$ (MRD $\mathrm{n}=29$, matched unrelated donor [MUD] $n=3$ ); autoHSCT, $n=20$; intensive chemotherapy, $\mathrm{n}=72$. The median time interval from diagnosis to achievement of CR1 was 1.1 months (range 0.36-4.11) with a trend $(P=.053)$ for a longer interval in patients who received an autoHSCT as postremission treatment (median 1.25 months) compared with those who received an alloHSCT (median 1.1 months) or chemotherapy (median 1.1 months). The median time interval from CR 1 to alloHSCT and autoHSCT was 4.0 months (range 0.8-6.5) and 2.3 months (range 0.4-6.2), respectively.

In total, 45 relapses ( 1 after alloHSCT, 5 after autoHSCT, and 39 after intensive chemotherapy) and 19 treatment-related deaths (7 after alloHSCT, 3 after autoHSCT, and 9 after intensive chemotherapy) after a median time measured from CR1 of 10.8 months (range 4.4-61) and 15 months (range 1.2-144) occurred. This leads to estimates of CIR and CID after 5 years of 3\% (SE 3\%) and 24\% (SE 8\%) for alloHSCT, 27\% (SE 11\%) and 13\% (SE 9\%) for autoHSCT, and 58\% (SE 6\%) and 10\% (SE 4\%) for intensive chemotherapy, respectively.

\section{Treatment after relapse}

After relapse, 41 patients received intensive reinduction therapy, 1 patient had repetitive cycles of subcutaneous azacitidine, and 3 patients were treated with supportive care only. The second CR rate (CR2) in all relapsed patients was 78\% (35 of 45) and in those receiving reinduction therapy (including azacitidine) $83 \%$ (35 of 42). Thirty-three patients received an alloHSCT (MRD $\mathrm{n}=11$,

Table 2. Abnormal karyotypes grouped according to leading aberrations

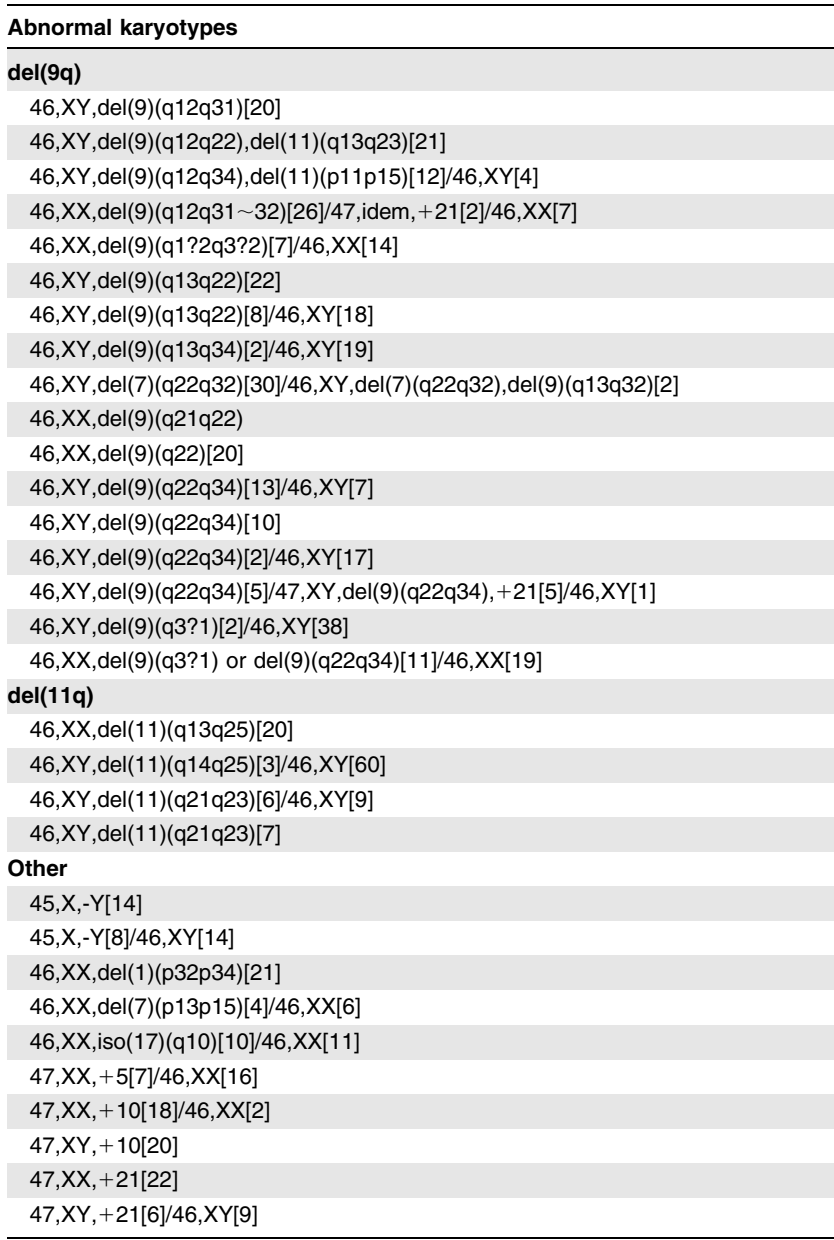



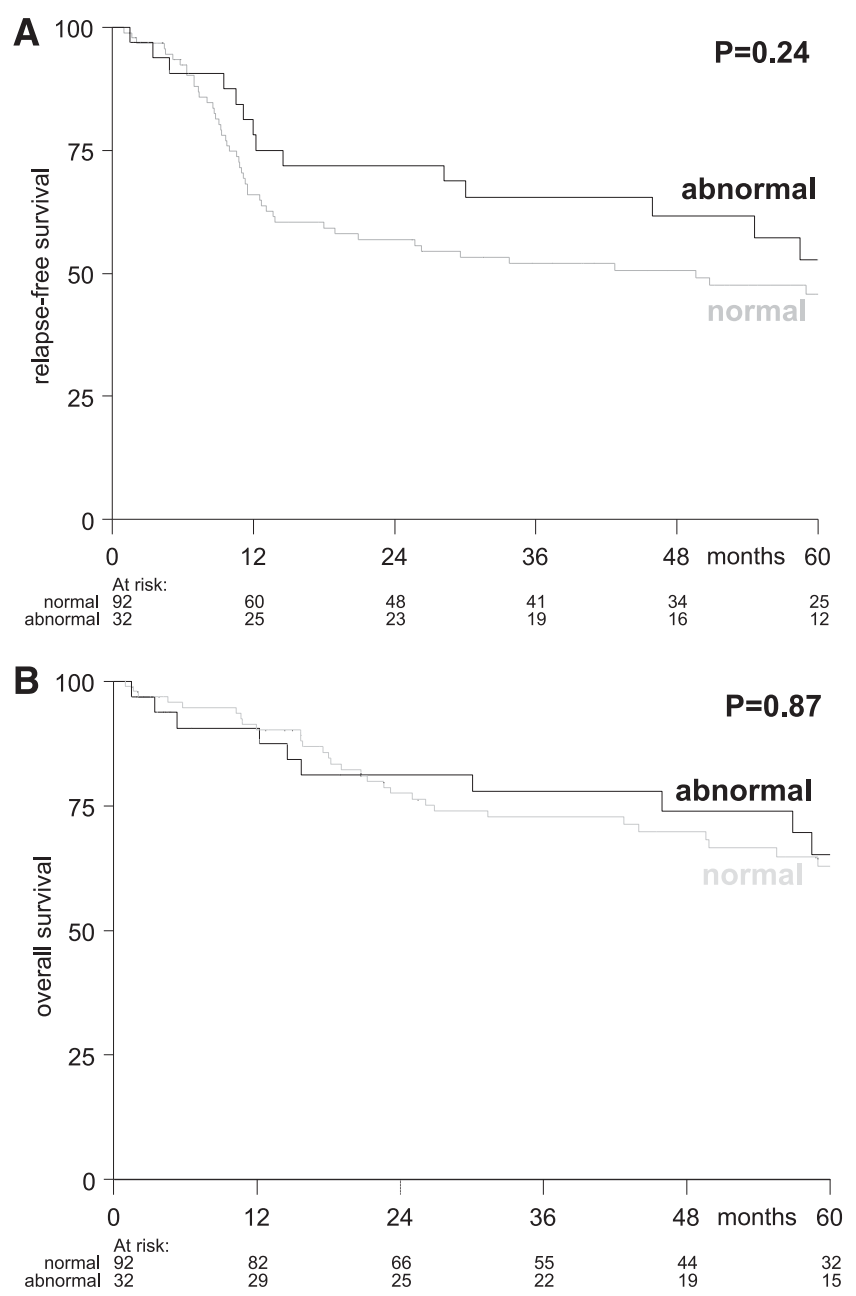

Figure 2. Influence of karyotype abnormalities on outcome. Kaplan-Meier plots for the endpoints (A) RFS and (B) OS according to the karyotype (normal vs abnormal).

MUD $\mathrm{n}=19$, haplo-identical donor $\mathrm{n}=3$ ) after reinduction therapy. At the time of alloHSCT, 28 patients achieved a second CR2 after reinduction therapy, 4 had refractory disease, and 1 patient who relapsed after alloHSCT in CR1 received a stem-cell boost from the same donor in CR2. Only 1 patient was treated with an autoHSCT after relapse.

\section{Survival analysis}

The median follow-up time of patients still alive at the date of last contact was 62 months. RFS and OS of the whole CEBPAdm patient cohort after 5 years were $48 \%$ (95\% CI, 38-57) and 63\% (95\% CI, 53-72), respectively. There was no difference in RFS $(P=.24)$ and OS $(P=.87)$ between patients exhibiting a normal karyotype and those with intermediate-risk karyotypes (Table 2 and Figure 2). Based on the Mantel-Byar test, including alloHSCT and autoHSCT applied in CR1 as time dependent variables, RFS is significantly superior in patients receiving an $\operatorname{HSCT}(P<.001)$, with significant differences in favor of alloHSCT and autoHSCT as compared with intensive chemotherapy $(P<.001$ and $P=.019$, respectively) (Figure 3A). Multivariable analysis based on the Andersen-Gill model including time-dependent postremission strategy, as well as pretreatment values (Table 1) of white blood cells, platelets, bone marrow (BM)-blast percentage, age, and karyotype (normal vs abnormal) revealed that alloHSCT (hazard ratio [HR] $0.23 ; P<.001$ ) and autoHSCT (HR 0.37; $P=.012$ ) applied in CR1 independently had a favorable prognostic impact regarding RFS (Table 3). However, apparently due to a high second CR rate after salvage therapy, the superior RFS after alloHSCT and autoHSCT did not translate into a better OS in univariable analysis $(P=.12)$ (Figure 3B) and multivariable analyses (Table 4).

In 45 relapsed patients, OS measured from the date of relapse was $46 \%$ (95\% CI, 30-60) after 3 years (Figure 4). All patients surviving more than 2 years after having first relapsed had undergone an alloHSCT $(n=15$, Figure 4$)$.

\section{Discussion}

This report focuses on the evaluation of the clinical impact in CEBPAdm patients of alloHSCT and autoHSCT in comparison with intensive consolidation therapy in CR1, as well as focusing on the impact of reinduction chemotherapy and alloHSCT after relapse. To this attempt, we report on 124 adults with AML and a normal karyotype or intermediate-risk karyotypes that harbor a CEBPAdm, and are aged $\leqslant 60$ years. Only 1 relapsed patient received an autoHSCT in second CR and, therefore, we were not able to evaluate the clinical impact of autoHSCT after relapse. Our results clearly show that adult AML patients with the CEBPAdm genotype significantly benefit from alloHSCT and autoHSCT in CR1 with respect to RFS.
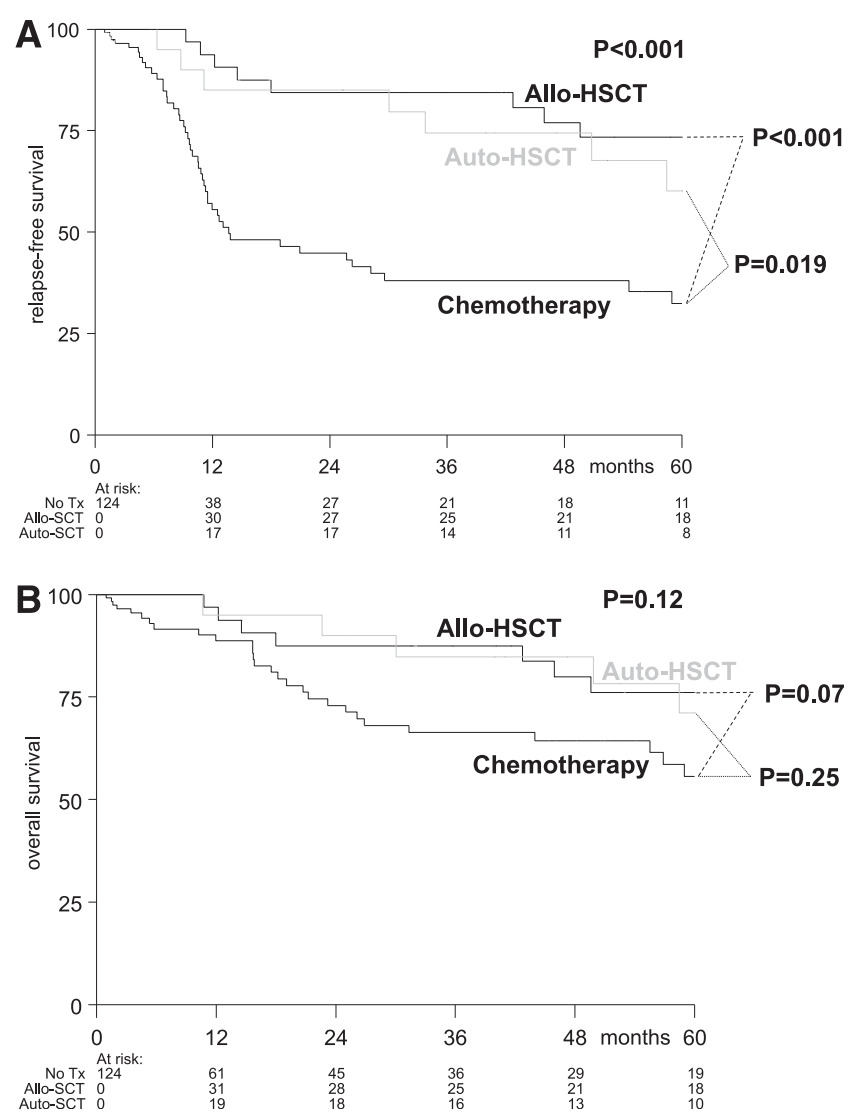

Figure 3. Influence of postremission treatment modality (alloHSCT, autoHSCT, chemotherapy) on RFS (A) and OS (B). Simon-Makuch plots for the endpoints (A) RFS and (B) OS according to type of postremission therapy. 
Table 3. Andersen-Gill model for the end point RFS

\begin{tabular}{lccc}
\hline & \multicolumn{3}{c}{ RFS } \\
\cline { 2 - 4 } Prognostic markers & HR & $\mathbf{9 5 \% ~ C l}$ & $\boldsymbol{P}$ \\
\hline Allogeneic HSCT & 0.23 & $0.11-0.51$ & $<.001$ \\
Autologous HSCT & 0.37 & $0.17-0.80$ & .012 \\
Log $_{10}($ WBC) & 1.40 & $0.77-2.56$ & .27 \\
Log $_{10}$ (platelets) & 0.81 & $0.40-1.66$ & .57 \\
$\%$ BM blasts (difference 10\%) & 0.94 & $0.82-1.08$ & .40 \\
Age (difference of 10 y) & 0.86 & $0.68-1.08$ & .20 \\
Abnormal karyotype & 0.73 & $0.38-1.40$ & .35 \\
\hline
\end{tabular}

WBC, white blood cell count.

In recent years, it has become apparent that the favorable prognosis of $C E B P A$ gene mutations largely depends on the presence of the $C E B P A \mathrm{dm}$ mutation type. At the molecular level AML with the $C E B P A \mathrm{dm}$ compared with AML with $C E B P A$ single mutation type is associated with a lower frequency of coexisting NPM1 mutations and FLT3 internal tandem duplications. ${ }^{6,8}$ Therefore, several investigators have recently suggested restricting the provisional WHO 2008 entity AML with CEBPA mutations to those with biallelic mutations. ${ }^{4-8}$ As a direct consequence, the incidence of this AML entity defined by $C E B P A d m$ decreases by about $40 \%{ }^{5,8}$ to a frequency of $3 \%$ to $6 \%$ of all AML cases. The low frequency of $C E B P A \mathrm{dm}$ explains why comparative analyses with regard to different postremission strategies, such as alloHSCT, autoHSCT, and intensive chemotherapy, have not been performed so far. Beside the recommended ELN risk category ${ }^{2}$ AML with CEBPA, we also included the group of intermediate-risk karyotypes into the analyses that includes approximately $30 \%$ of patients with chromosomal abnormalities, in particular interstitial deletion $9 \mathrm{q}^{28}$ and $11 \mathrm{q}$ (Table 2) instead of only patients with AML exhibiting a normal karyotype. This approach is supported by the similar favorable outcome in AML with CEBPAdm with and without normal karyotype in univariable and multivariable analyses presented here (Figure 2). Thus, these data add evidence that AML with CEBPAdm may be regarded as a distinctive AML entity irrespective of additional chromosomal abnormalities categorized within the cytogenetically defined intermediate-risk group. ${ }^{2}$

In the current analyses, we started with a total cohort of 5724 patients from which 5147 had a karyotype and, of those, the CEBPA mutational status in 2983 was available. This large cohort was required to finally achieve a sufficiently high number of 124 AML patients with $C E B P A \mathrm{dm}$ in CR1 representing the basis of our analyses. This approach underlines that large cooperative intergroup meta-analyses are warranted to evaluate treatment effects with acceptable statistical power in rare but clinically, highly relevant patient subsets.

Our patients were treated in 7 different treatment trials, in part, with changing therapeutic concepts over time. Thus, it is impossible to apply the rigorous statistical standards for postremission treatment allocation such as up-front or the so-called genetic randomization. Instead, we applied statistical methods that have all in common, that group allocation is implemented as a dynamic process over time with a transition from the no-transplant group to the alloHSCT or autoHSCT groups at the time point of HSCT. This approach reduces the time-to-treatment bias and provides a solid statistical methodology in situations in which simple Kaplan-Meier plots and log-rank tests, as well as simple Cox regression models, are no longer valid. However, to further reduce selection bias toward HSCT in CR1, our univariable comparisons were complemented by multivariable Andersen-Gill regression models addressing the time-to-treatment bias $^{27}$ again by including important pretreatment characteristics.

By using methods that adjust for the time from CR to consolidation, we were able to show a clear superior RFS $(P<.001)$ in patients who received an alloHSCT or an autoHSCT in CR1 of $73 \%$ (95\% CI, 54-86) and 60\% (95\% CI, 33-79) after 5 years, respectively. These survival rates compare favorably to the RFS of $32 \%$ (95\% CI, 21-45) in patients receiving intensive chemotherapy only. A similarly good outcome has also been reported for other AML entities categorized into the favorable ELN risk group, such as core binding factor AML (CBF-AML), including AML with inv(16) or $\mathrm{t}(16 ; 16)$ and AML with $\mathrm{t}(8 ; 21)$ after both alloHSCT and autoHSCT. ${ }^{29,30}$ However, our results suggest that RFS after intensive chemotherapy is substantially lower for AML with CEBPAdm as compared with that of CBF-AML. ${ }^{31,32}$ Nevertheless, due to a high second CR rate in reinduced relapsed patients with more than $80 \%$ and a high proportion of patients proceeding to an alloHSCT after relapse, the high relapse rate in the chemotherapy subgroup did not translate into a significant inferior OS. In fact, the high second CR rate and favorable survival after relapse observed in the study reported here are comparable to the survival probabilities observed in AML with inv(16). ${ }^{31,32}$ Based on these data, AML with CEBPAdm and AML with inv(16) or $\mathrm{t}(16 ; 16)$ appear as 2 well-defined exceptions from the general notion that after relapse a second $\mathrm{CR}$ is rarely achieved. ${ }^{33}$ Therefore, instead of applying alloHSCT as the compelling option in $\mathrm{CR} 1$, an alternative and not unreasonable strategy would be to postpone the alloHSCT in CR1 and keep the option of alloHSCT for salvage for the restricted fraction of patients after relapse. ${ }^{33,34}$ Indeed, our data supports both strategies, alloHSCT or autoHSCT in CR vs intensive chemotherapy as consolidation in CR1, and reinduction followed by alloHSCT in case of relapse. Of note, the good results in our study with autoHSCT are paralleled by those obtained in the core-binding factor $\mathrm{AML}^{29,30}$ indicating a specific chemo-sensitivity of these AMLs with favorable risk according to the ELN recommendations ${ }^{2}$ to dose escalation during consolidation therapy in CR1. Patients have to be well informed about the risks and consequences of alloHSCT and autoHSCT in CR1 regarding (1) short-term ${ }^{35}$ and long-term ${ }^{36}$ physical and psychological impairment; (2) infertility and a higher rate of treatment-related mortality (eg, for alloHSCT, we observed $24 \%$ at 5 years in our cohort); and (3) increased rates of transplantation-related morbidity and mortality for alloHSCT when the latter is performed after relapse. ${ }^{37}$ Besides the survival considerations, there are other various arguments that would favor the choice of an autoHSCT in CR1 as compared with alloHSCT. Given the nearly identical RFS and OS rates after alloHSCT and autoHSCT, the focus of outcome evaluations can be broadened to consider quality of life aspects and late effects after transplantation, as well as health economics, with a significantly better quality of life and

Table 4. Andersen-Gill model for the endpoint OS

\begin{tabular}{lccc}
\hline & \multicolumn{3}{c}{ RFS } \\
\cline { 2 - 4 } Prognostic markers & HR & $\mathbf{9 5 \% - C l}$ & $\boldsymbol{P}$ \\
\hline Allogeneic HSCT & 0.50 & $0.21-1.17$ & .11 \\
Autologous HSCT & 0.57 & $0.23-1.40$ & .22 \\
Log $_{10}($ WBC) & 1.34 & $0.64-2.80$ & .44 \\
Log $_{10}$ (platelets) & 0.95 & $0.40-2.26$ & .91 \\
\% BM blasts (difference 10\%) & 1.04 & $0.87-1.24$ & .69 \\
Age (difference of 10 y) & 1.08 & $0.82-1.42$ & .59 \\
Abnormal karyotype & 1.14 & $0.55-2.34$ & .73 \\
\hline
\end{tabular}

WBC, white blood cell count. 


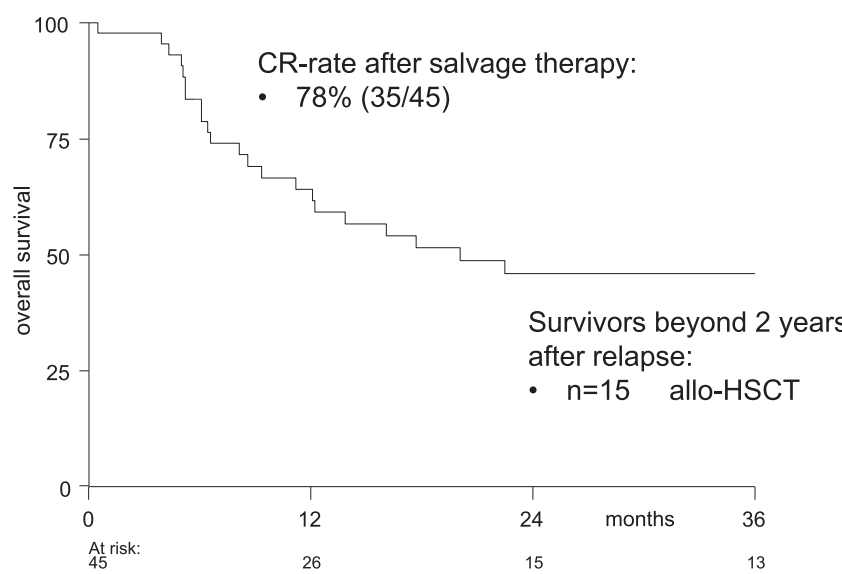

Figure 4. Outcome of patients after relapse. Simon-Makuch plot for the end point OS, second CR rate after salvage remission induction therapy and treatment details for patients surviving longer than 2 years.

fewer late effects after autoHSCT compared with alloHSCT, ${ }^{38}$ whereas data on health economics are very system specific. ${ }^{39}$

In summary, our data provide novel clinical information that may be useful for refining the WHO 2008 classification and the ELN risk categorization for the provisional entity AML with CEBPAdm in that beyond normal karyotype, all intermediate-risk cytogenetics should be included. From a clinical perspective alloHSCT and autoHSCT performed in CR1 were associated with comparatively excellent RFS and OS, whereas the reduced rate of RFS in patients receiving consolidation with intensive chemotherapy could be made up after relapse by a high rate of second CR followed by alloHSCT. Thus, the marker CEBPAdm develops with respect to RFS to a predictive marker indicating superior RFS after autoHSCT and alloHSCT, but remains a prognostic marker with respect to OS. The pros and cons of alloHSCT and autoHSCT during CR1 or, as an alternative option, alloHSCT after relapse, have to be carefully considered and discussed with the patients with possible individual adaptation of treatment recommendations while taking into account the patient's personal context.

\section{Acknowledgments}

The authors thank Jasper Koenders and Francois Kavelaars for their contribution in the collection of the data.

This work was supported by grants from the Center for Translational Molecular Medicine and by Else Kröner-FreseniusStiftung grant P38/05//A49/05//F03, the Network of Competence Acute and Chronic Leukemias (01GI9981) and the Bundesministerium für Bildung und Forschung, Germany (01KG0605) ("IPD-metaanalysis: a model-based hierarchical prognostic system for adult patients with AML").

\section{Authorship}

Contribution: R.F.S., E.T., B.L., Y.v.N., H.D., K.D., and R.D. provided concept and design; R.F.S., E.T., J.K., A.G., V.G., P.P., G.G., A.K., K.G., P.V., J.J.C., G.J.O., J.K., U.S., E.V., H.D., R.D., K.D., and B.L. provided study materials or patients; R.F.S., E.T., Y.v.N., J.K., A.G., V.G., P.P., G.G., A.K., G.H., K.G., P.V., J.J.C., G.J.O., J.K., U.S., E.V., H.D., R.D., K.D., B.L. collected and assembled the data; R.F.S., E.T., B.L., Y.v.N., H.D., K.D. provided data analysis and interpretation; R.F.S., E.T., B.L., Y.v.N., H.D., K.D. wrote the manuscript; R.F.S., E.T., Y.v.N., J.K., A.G., L.B., V.G., P.P., A.C., G.G., A.K., G.H., K.G., E.V., J.K., U.S., J.P., T.P., J.M., G.J.O., R.D., H.D., J.J.C., K.D., B.L. gave final approval of the manuscript; and K.D. and B.L. were both seniors authors of the manuscript.

Conflict-of-interest disclosure: The authors declare no competing financial interests.

Correspondence: Richard F. Schlenk, Department of Internal Medicine III, University Hospital Ulm, Albert Einstein Allee 23, 89081 Ulm, Germany; e-mail: richard.schlenk@uniklinik-ulm.de; and Bob Löwenberg, Department of Hematology, Erasmus University Medical Center, POB 2040, 3000CA Rotterdam, The Netherlands; e-mail: b.lowenberg@erasmusmc.nl.

\section{References}

1. Swerdlow SH, Campo E, Harris NL, et al. WHO classification of tumours of the haematopoietic and lymphoid tissues. 4th ed. Geneva, Switzerland: WHO Press; 2008.

2. Döhner H, Estey EH, Amadori S, et al; European LeukemiaNet. Diagnosis and management of acute myeloid leukemia in adults: recommendations from an international expert panel, on behalf of the European LeukemiaNet. Blood. 2010;115(3):453-474.

3. Pabst T, Eyholzer M, Fos J, Mueller BU. Heterogeneity within AML with CEBPA mutations; only CEBPA double mutations, but not single CEBPA mutations are associated with favourable prognosis. Br J Cancer. 2009;100(8):1343-1346.

4. Wouters BJ, Löwenberg B, Erpelinck-Verschueren CA, van Putten WL, Valk PJ, Delwel R. Double CEBPA mutations, but not single CEBPA mutations, define a subgroup of acute myeloid leukemia with a distinctive gene expression profile that is uniquely associated with a favorable outcome. Blood. 2009;113(13):3088-3091.

5. Dufour A, Schneider F, Metzeler $\mathrm{KH}$, et al. Acute myeloid leukemia with biallelic CEBPA gene mutations and normal karyotype represents a distinct genetic entity associated with a favorable clinical outcome. J Clin Oncol. 2010; 28(4):570-577.

6. Green CL, Koo KK, Hills RK, Burnett AK, Linch DC, Gale RE. Prognostic significance of CEBPA mutations in a large cohort of younger adult patients with acute myeloid leukemia: impact of double CEBPA mutations and the interaction with FLT3 and NPM1 mutations. J Clin Oncol. 2010; 28(16):2739-2747.

7. Hou HA, Lin LI, Chen CY, Tien HF. Reply to 'Heterogeneity within AML with CEBPA mutations; only CEBPA double mutations, but not single CEBPA mutations are associated with favorable prognosis'. Br J Cancer. 2009;101(4):738-740.

8. Taskesen E, Bullinger L, Corbacioglu A, et al. Prognostic impact, concurrent genetic mutations, and gene expression features of AML with CEBPA mutations in a cohort of 1182 cytogenetically normal AML patients: further evidence for CEBPA double mutant AML as a distinctive disease entity. Blood. 2011;117(8): 2469-2475.

9. Bereshchenko O, Mancini E, Moore S, et al. Hematopoietic stem cell expansion precedes the generation of committed myeloid leukemia-initiating cells in C/EBPalpha mutant AML. Cancer Cell. 2009;16(5):390-400.
10. Schlenk RF, Döhner K, Krauter J, et al; German-Austrian Acute Myeloid Leukemia Study Group. Mutations and treatment outcome in cytogenetically normal acute myeloid leukemia. N Engl J Med. 2008;358(18):1909-1918.

11. Fröhling S, Schlenk RF, Stolze I, et al. CEBPA mutations in younger adults with acute myeloid leukemia and normal cytogenetics: prognostic relevance and analysis of cooperating mutations. J Clin Oncol. 2004;22(4):624-633.

12. Preudhomme $\mathrm{C}$, Sagot $\mathrm{C}$, Boissel N, et al; ALFA Group. Favorable prognostic significance of CEBPA mutations in patients with de novo acute myeloid leukemia: a study from the Acute Leukemia French Association (ALFA). Blood. 2002;100(8):2717-2723.

13. Schlenk RF, Döhner K, Kneba M, et al; German-Austrian AML Study Group (AMLSG). Gene mutations and response to treatment with all-trans retinoic acid in elderly patients with acute myeloid leukemia. Results from the AMLSG Trial AML HD98B. Haematologica. 2009;94(1):54-60.

14. Mrózek K, Marcucci G, Nicolet D, et al. Prognostic significance of the European LeukemiaNet standardized system for reporting cytogenetic and molecular alterations in adults with acute myeloid leukemia. J Clin Oncol. 2012;30(36):4515-4523. 
15. Löwenberg B, van Putten W, Theobald M, et al Dutch-Belgian Hemato-Oncology Cooperative Group; Swiss Group for Clinical Cancer Research. Effect of priming with granulocyte colony-stimulating factor on the outcome of chemotherapy for acute myeloid leukemia. N Engl J Med. 2003;349(8):743-752.

16. Pabst $\mathrm{T}$, Vellenga $\mathrm{E}$, van Putten $\mathrm{W}$, et al Dutch-Belgian Hemato-Oncology Cooperative Group (HOVON); German AML Study Group (AMLSG); Swiss Collaborative Group for Clinical Cancer Research (SAKK). Favorable effect of priming with granulocyte colony-stimulating factor in remission induction of acute myeloid leukemia restricted to dose escalation of cytarabine. Blood. 2012;119(23):5367-5373.

17. Vellenga E, van Putten W, Ossenkoppele GJ et al; Dutch-Belgian Hemato-Oncology Cooperative Group (HOVON); Swiss Group for Clinical Cancer Research Collaborative Group (SAKK). Autologous peripheral blood stem cell transplantation for acute myeloid leukemia. Blood. 2011;118(23):6037-6042.

18. Löwenberg B, Pabst T, Vellenga $E$, et al; Dutch-Belgian Cooperative Trial Group for Hemato-Oncology (HOVON) and Swiss Group for Clinical Cancer Research (SAKK) Collaborative Group. Cytarabine dose for acute myeloid leukemia. N Engl J Med. 2011;364(11): 1027-1036.

19. Schlenk RF, Benner A, Hartmann F, et al; AML Study Group UIm (AMLSG ULM). Risk-adapted postremission therapy in acute myeloid leukemia: results of the German multicenter AML HD93 treatment trial. Leukemia. 2003;17(8):1521-1528.

20. Schlenk RF, Döhner K, Mack S, et al. Prospective evaluation of allogeneic hematopoietic stem-cell transplantation from matched related and matched unrelated donors in younger adults with high-risk acute myeloid leukemia: German-Austrian trial AMLHD98A. J Clin Oncol. 2010;28(30):4642-4648.

21. Care RS, Valk PJ, Goodeve AC, et al. Incidence and prognosis of C-KIT and FLT3 mutations in core binding factor (CBF) acute myeloid leukaemias. Br J Haematol. 2003;121(5):775-777.

22. Valk PJ, Bowen DT, Frew ME, Goodeve AC Löwenberg B, Reilly JT. Second hit mutations in the RTK/RAS signaling pathway in acute myeloid leukemia with inv(16) Haematologica. 2004;89(1): 106.

23. Verhaak RG, Goudswaard CS, van Putten W, et al. Mutations in nucleophosmin (NPM1) in acute myeloid leukemia (AML): association with other gene abnormalities and previously established gene expression signatures and their favorable prognostic significance. Blood. 2005 106(12):3747-3754

24. Gray RJ. A class of k-sample tests for comparing the cumulative incidence of a competing risk. Ann Stat. 1988;16(3):1141-1154

25. Simon R, Makuch RW. A non-parametric graphical representation of the relationship of an event: application to responser versus nonresponder bias. Stat Med. 1984;3:35-44.

26. Mantel N, Byar D. 'Evaluation of response-time data involving transient states: An illustration using heart transplant data'. J Am Stat Assoc. 1974;69:81-86.

27. Andersen P, Gill RD. Cox's regression model for counting processes: A large sample study. Ann Stat. 1982;10:1100-1120.

28. Fröhling S, Schlenk RF, Krauter J, et al. Acute myeloid leukemia with deletion $9 q$ within a noncomplex karyotype is associated with CEBPA loss-of-function mutations. Genes Chromosomes Cancer. 2005;42(4):427-432.

29. Gorin NC, Labopin M, Frassoni F, et al. Identical outcome after autologous or allogeneic genoidentical hematopoietic stem-cell transplantation in first remission of acute myelocytic leukemia carrying inversion 16 or $t(8 ; 21)$ : a retrospective study from the European Cooperative Group for Blood and Marrow Transplantation. J Clin Oncol. 2008;26(19): 3183-3188

30. Kuwatsuka Y, Miyamura K, Suzuki R, et al. Hematopoietic stem cell transplantation for core binding factor acute myeloid leukemia: $t(8 ; 21)$ and inv(16) represent different clinical outcomes. Blood. 2009;113(9):2096-2103.

31. Schlenk RF, Benner A, Krauter J, et al. Individual patient data-based meta-analysis of patients aged 16 to 60 years with core binding factor acute myeloid leukemia: a survey of the German Acute
Myeloid Leukemia Intergroup. J Clin Oncol. 2004 22(18):3741-3750.

32. Marcucci G, Mrózek K, Ruppert AS, et al. Prognostic factors and outcome of core binding factor acute myeloid leukemia patients with $t(8 ; 21)$ differ from those of patients with inv(16): a Cancer and Leukemia Group B study. J Clin Oncol. 2005;23(24): 5705-5717.

33. Forman SJ, Rowe JM. The myth of the second remission of acute leukemia in the adult. Blood. 2013;121(7):1077-1082.

34. Burnett AK, Goldstone A, Hills RK, et al. Curability of patients with acute myeloid leukemia who did not undergo transplantation in first remission [published online ahead of print February 25, 2013]. J Clin Oncol.

35. Watson M, Buck G, Wheatley K, et al. Adverse impact of bone marrow transplantation on quality of life in acute myeloid leukemia patients; analysis of the UK Medical Research Council AML 10 trial. The UK Medical Research Council AML 10 trial. Eur J Cancer. 2004;40: 971-978.

36. Messerer D, Engel J, Hasford J, et al; German AML Intergroup. Impact of different postremission strategies on quality of life in patients with acute myeloid leukemia. Haematologica. 2008;93(6):826-833.

37. Cornelissen JJ, Gratwohl A, Schlenk RF, et al. The European LeukemiaNet AML Working Party consensus statement on allogeneic HSCT for patients with $\mathrm{AML}$ in remission: an integrated-risk adapted approach. Nat Rev Clin Oncol. 2012 9(10):579-590

38. Khera N, Storer B, Flowers ME, et al. Nonmalignant late effects and compromised functional status in survivors of hematopoietic cell transplantation. J Clin Oncol. 2012;30(1):71-77.

39. Ashfaq K, Yahaya I, Hyde C, et al. Clinical effectiveness and cost-effectiveness of stem cell transplantation in the management of acute leukaemia: a systematic review. Health Technol Assess. 2010;14(54):iii-iv, ix-xi, $1-141$ 